\title{
Comparison of the selective targeting efficacy of Salmonella typhimurium A1-R and VNP20009 on the Lewis lung carcinoma in nude mice
}

\author{
Yong Zhang ${ }^{1}$, Nan Zhang ${ }^{1}$, Ming Zhao ${ }^{1}$ and Robert M. Hoffman ${ }^{1,2}$ \\ ${ }^{1}$ AntiCancer, Inc., San Diego, California, USA \\ 2 Department of Surgery, University of California San Diego, California, USA \\ Correspondence to: Robert M. Hoffman, email: all@anticancer.com \\ Keywords: lung cancer, Salmonella typhimurium A1-R, VNP20009, GFP, RFP
}

Received: December 16,2014 Accepted: January 03,2015 Published: January 21, 2015

This is an open-access article distributed under the terms of the Creative Commons Attribution License, which permits unrestricted use, distribution, and reproduction in any medium, provided the original author and source are credited.

\section{ABSTRACT}

Salmonella typhimurium A1-R is auxotrophic for arg and leu, which attenuates growth in normal tissue but allows high tumor targeting and virulence. A1-R is effective against metastatic human prostate, breast, and pancreatic cancer as well as osteosarcoma, fibrosarcoma, and glioma in clinically-relevant mouse models. VNP20009 is also a genetically-modified strain of Salmonella typhimurium that has been tested in Phase I clinical trials, but is more attenuated than $S$. typhimurium A1-R and in addition of multiple amino-acid auxotrophs, is purine auxotropic with the purI mutation. In the present study, mouse Lewis lung carcinoma-bearing nude mouse models were treated with S. typhimurium A1-R or VNP20009. S. typhimurium A1-R and VNP20009 were both eliminated from the liver and spleen approximately 3-5 days after administration via the tail vein. However, A1-R showed higher tumor targeting and inhibited the Lewis lung carcinoma to a greater extent than VNP20009, with less body weight loss. The mice tolerated $S$. typhimurium A1-R to at a least 2-fold higher dose than VNP20009 when the bacteria were administered iv. The results of the present study suggest that $S$. typhimurium A1-R has greater clinical potential than VNP20009.

\section{INTRODUCTION}

For more than 200 years, cancers have been observed to regress following acute infections, mostly streptococcal [1]. In the late 19th and early 20th centuries. Coley infected cancer patients with Streptococcus pyrogenes and later treated the patients with extracts of the bacteria, which became known as Coley's toxins. Coley had remarkable results with both the bacteria and the toxins [2]. However bacterial therapy of cancer stopped after Coley's death in 1936 [2].

Recently, there has been intense renewed interest to develop bacterial therapy of cancer [2-4]. The barriers in tumors for standard therapy such as hypoxia, acidic $\mathrm{pH}$, disorganized vascular architecture, are beneficial for bacteria to target cancer [3].

One approach to bacterial therapy of cancer is to use anaerobic bacteria such as Bifidobacterium [5] and Clostridium [6] which replicate in necrotic areas of tumors. These anaerobic bacteria cannot grow in viable tumor tissue, which restricts their efficacy. In addition, obligate anaerobic bacteria may be limited to intratumor injection which would preclude their use for metastatic cancer.

Recently a human patient with metastatic leiomyosarcoma was treated by intratumoral injection of Clostridium novyi (C. novyi)-NT spores which reduced the tumor within and surrounding the bone [7]. However other tumor deposits in the patient were not affected.

$S$. typhimurium is a facultative anaerobe and therefore unlike anaerobe bacteria can infect viable portions of tumors as well as necrotic areas. The VNP20009 strain of S. typhimurium, attenuated with purine and other auxotrophic mutations, has been previously used for cancer therapy [8]. When NP20009 were inoculated i.p. into C57B6 mice bearing the B16F10 melanoma, it suppressed tumor growth and prolonged average survival to as much as twice that of untreated 
mice [9].

VNP20009 is attenuated with a lipid A-mutation $(m s b B)$, purine auxotrophy (purl) and amino acid auxotrophs [8]. In a Phase I clinical trial on patients with metastatic melanoma and renal-cell carcinoma, VNP20009 was safely administered, but poorly colonized the patients' tumors, perhaps because it was overattenuated [10]. At the highest tolerated dose, some tumor colonization was observed [10].

Another strain of S. typhimurium, A1-R, has been developed by our laboratory, which has greatly increased antitumor efficacy. S. typhimurium A1-R is auxotrophic only for leu-arg which prevents it from mounting a continuous infection in normal tissues. $S$. typhimurium A1-R has no other attenuating mutations as does VNP20009. A1-R was able to eradicate primary and metastatic tumors in monotherapy in nude mouse models of prostate, breast, ovarian and pancreatic cancer, as well as sarcoma and glioma [11-19]. S. typhimurium A1-R also greatly inhibited bone and brain metastasis of breast cancer in orthotopic mouse models [20,21]. Tumors with a high degree of vascularity were more sensitive to $S$. typhimurium A1-R, and vascular destruction appears to play a role in S. typhimurium A1-R antitumor efficacy [22].

The present study compares $S$. typhimurium A1-R and VNP20009 for anti-tumor efficacy in a nude mouse model of highly aggressive lung cancer.

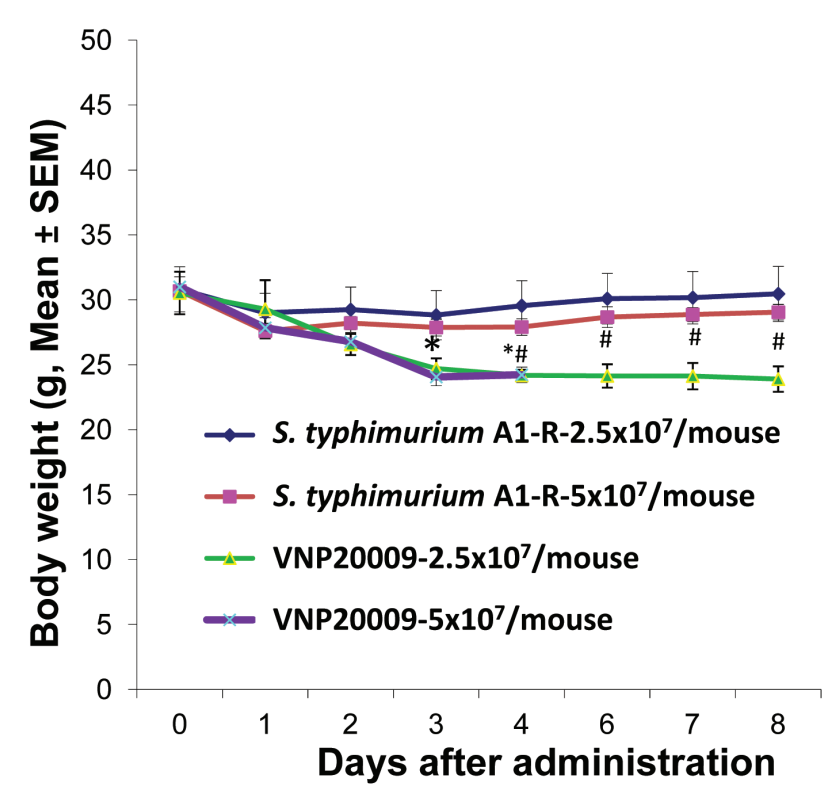

\section{RESULTS AND DISCUSSION}

\section{Comparison of toxicity of $S$. typhimurium A1-R and VNP20009}

There was lower toxicity of $S$. typhimurium A-1R in nude mice compared to $S$. typhimurium VNP20009. Treatment with $S$. typhimurium A1-R resulted in less body weight loss than with VNP20009 $(p=<0.05)$ (Fig. 1A). There was prolonged survival in mice treated with $S$. typhimurium A1-R as compared to the non-tumor-bearing mice treated with S. typhimurium VNP20009 $(p=0.005)$ (Fig. 1B).

There were less hemorrhagic spots on the skin and liver in mice treated with $S$. typhimurium A1-R than VNP20009 (Figure 2). Bleeding foci were found in the liver on day 3 after bacteria injection. However, VNP20009 has more bleeding foci on the liver than in $S$. typhimurium A1-R-treated mice $(p<0.05)$.

\section{Comparison of distribution of $S$. typhimurium A1-R and VNP20009 in tumor, liver, spleen, and blood}

When the average tumor volume reached approximately $70 \mathrm{~mm}^{3}$, S. typhimurium A1-R (1x10 $\mathrm{CFU})$ or VNP-20009 (1x107 CFU) were injected into the

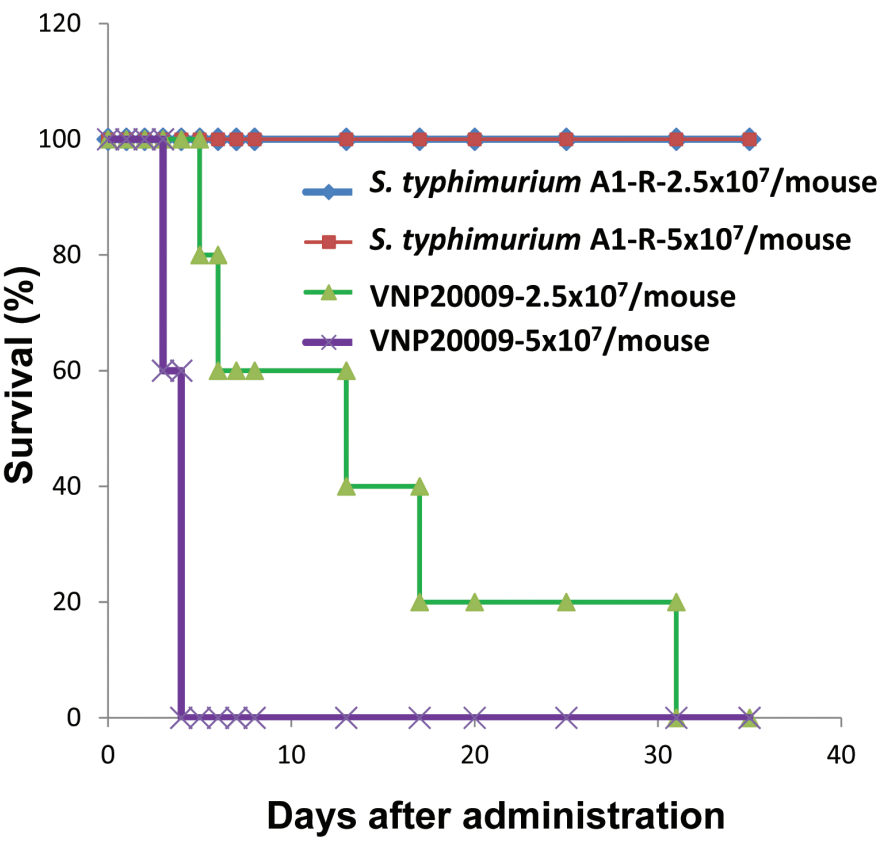

Figure 1: Comparison of body weight and survival of nude mice without tumors after $S$. typhimurium A1-R or VNP20009 i.v. administration. Female nude mice, aged 6 weeks, were injected iv with S. typhimurium A1-R or VNP20009 at different doses in 100 $\mu 1$ PBS. Body weight was monitored using an electronic scale. A: Body weight curve comparing S. typhimurium A1-R and VNP20009 at both doses $(p<0.05)$. B: Kaplan Meir survival curve. $\mathrm{n}=5$ mice for each group comparing S. typhimurium A1-R and VNP20009 at both doses $(p<0.05)$. 

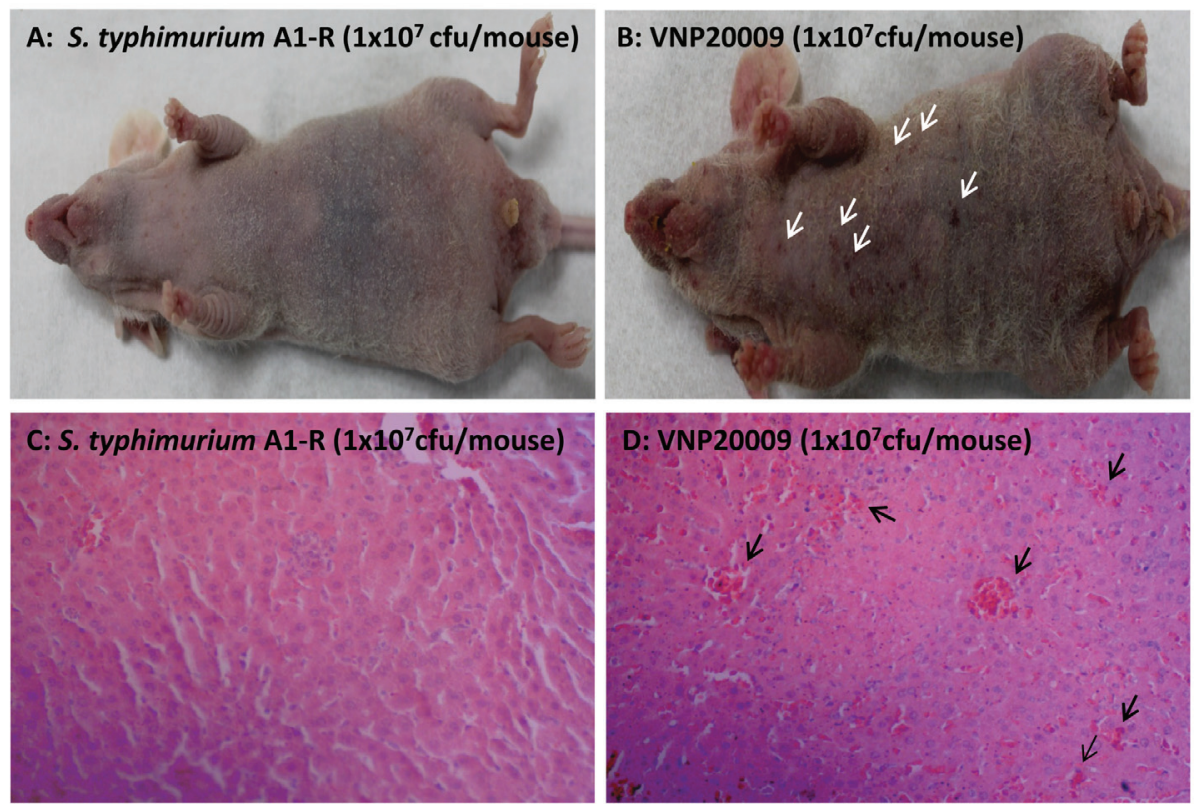

Figure 2: Comparison of overt toxicity of $S$. typhimurium A1-R and VNP20009. A: S. typhimurium A1-R treated mice had few hemorrhagic spots on the skin. B: VNP20009 treated mice had more hemorrhagic spots on the skin (white arrows). C: S. typhimurium A1-R treated mice had less bleeding foci on the liver on day 3 after bacteria infection. D: VNP20009 treated mice had more bleeding foci on the liver on the day 3 than $S$. typhimurium A1-R after bacteria infection (black arrows) $(p<0.05)$.

A
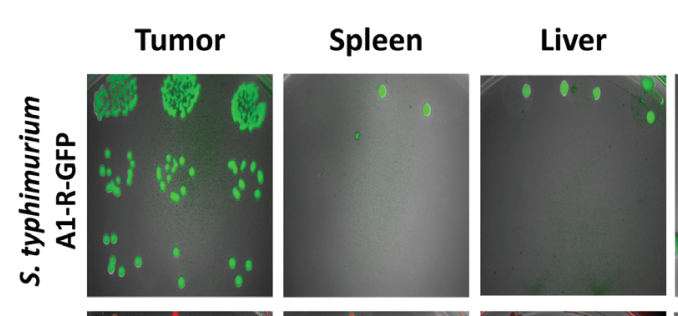

Blood
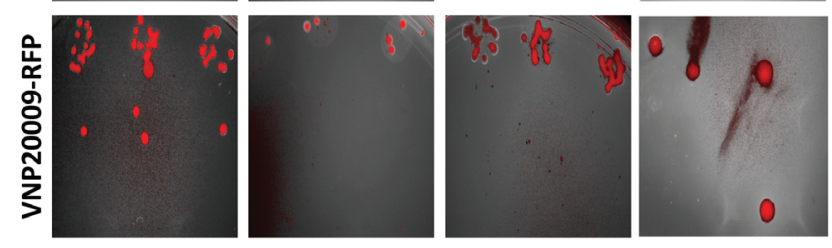

B

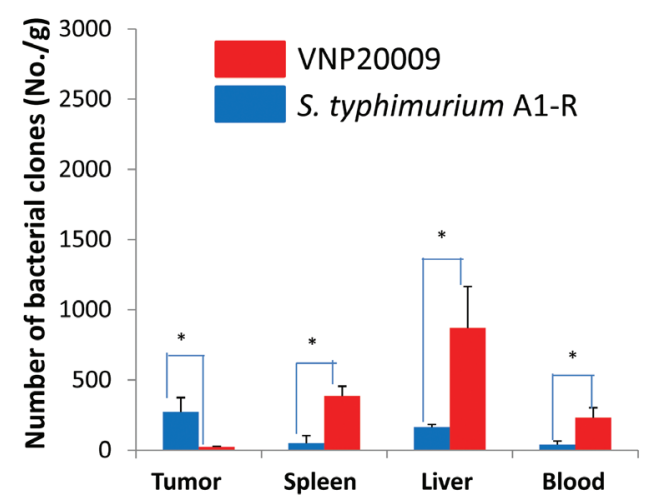

Figure 3: Comparison of tissue distribution of $S$. typhimurium A1-R and VNP20009 in tumor and normal tissues. When the average tumor volume reached approximately $70 \mathrm{~mm}^{3}$, S. typhimurium A1-R (1x10 CFU) or VNP-20009 (1x10 $\left.{ }^{7} \mathrm{CFU}\right)$ were injected into the tail vein. Tissues were removed 6 days after bacteria administration. Bacteria were isolated from the tumor and organs and cultured in LB agar $(* p<0.05)$. 
tail vein one time. Tissues were removed 6 days after bacteria administration. Bacteria were isolated from the tumor and organs and cultured in LB agar. Both strains selectively targeted the tumor with greater targeting by $S$. typhimurium A1-R than VNP20009. VNP20009 had more bacteria in the liver and spleen and blood $(p<0.05)$ (Figure $3)$.

\section{Comparison of efficacy of $S$. typhimurium A1-R and VNP20009}

S. typhimurium A1-R reduced tumor growth to a greater extent than VNP20009 ( $p<0.05)$ (Figure 4A). On day 10 , a significantly lower tumor burden in mice treated with $S$. typhimurium A1-R than mice treated with VNP20009 was observed. S. typhimurium A1-R-treated mice had a tumor weight $(0.594 \mathrm{~g} \pm 0.23)$ which was lower than with VNP20009-treated mice $(1.378 \mathrm{~g} \pm 0.51)$
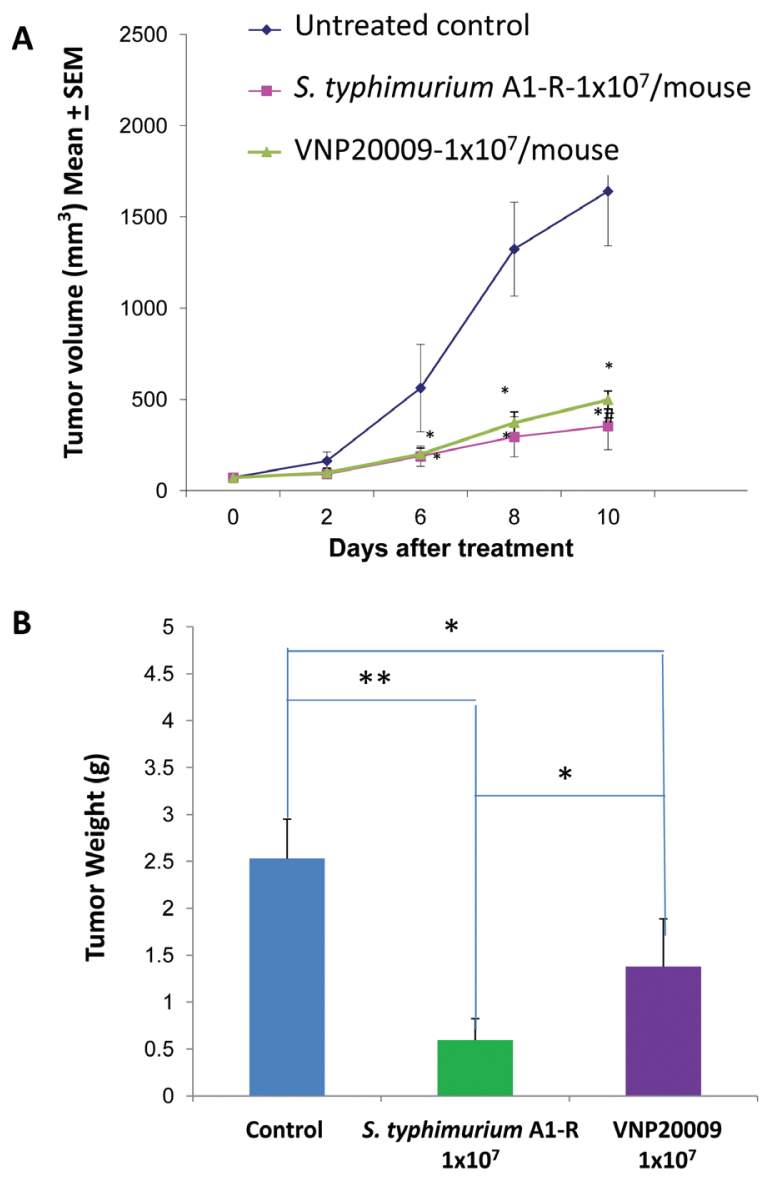

Figure 4: Comparison of efficacy of $S$. typhimurium A1-R or VNP20009 on the Lewis lung cancer. When the average tumor volume reached approximately $70 \mathrm{~mm}^{3}, S$.

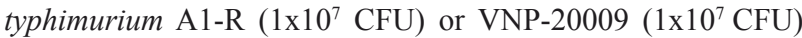
were injected into the tail vein once a week. Primary tumor sizes were measured with a caliper. A: Tumor growth curve. B: Tumor weight in S. typhimurium A1-R- and VNP20009-treated mice, as compared to control mice $(* p<0.05 ; * *<<0.01)$ at day 10 after treatment. $\mathrm{n}=5$ mice for each group. $(p<0.05)$. Control mice had a tumor weight of $(2.53 \mathrm{~g} \pm$ $0.42)$ which was greater compared to VNP20009-treated mice $(p<0.05)$ or $S$. typhimurium A1-R-treated mice $(\mathrm{p}<0.01)$ (Figure 4B).

\section{CONCLUSIONS}

S. typhimurium A1-R had lower toxicity in nude mice compared to $S$. typhimurium VNP20009. S. typhimurium A1-R and S. typhimurium VNP20009 both inhibited growth of the Lewis lung cancer in nude mouse subcutaneous models. However, S. typhimurium A1-R was more efficacious than S. typhimurium VNP20009 on tumor growth inhibition.

The results of the present study indicate $S$. typhimurium A1-R should enter clinical trials as soon as possible. Better results with $S$. typhimurium A1-R than VNP20009 should be expected since $S$. typhimurium A1-R is more virulent against the tumor and less toxic against the host. Better results with $S$. typhimurium A1-R than C. novyi-NT should be expected since $S$. typhimurium A1-R can be effectively administered systemically and C. novyi-NT seems limited to intratumor administration, possibly precluding it from targeting metastatic disease. A promising application of $S$. typhimurium A1-R is to decoy cancer cells within tumor to divide and thereby become sensitive to chemotherapy [23].

The bacterial tumor targeting described in the present report can take advantage of previously developed tumor targeting strategies [24-31].

\section{MATERIALS AND METHODS}

\section{Cells}

Lewis lung carcinoma cells were maintained in RPMI 1640 supplemented with 10\% fetal bovine serum, $2 \mathrm{mM}$ glutamine (Gibco-BRL, Life Technologies, Inc., Carlsbad, CA). The cell line was cultured at $37^{\circ} \mathrm{C}$ in a $5 \%$ incubator. RFP vector production and transduction of the Lewis lung cancer cell line were performed as previously published [32-34]. The RFP (DsRed-2) gene (Clontech Laboratories, Palo Alto, CA) was inserted in the retroviralbased mammalian expression vector pLNCX (Clontech) to form the pLNCX-DsRed-2 vector as previously described [32-34]. For RFP gene transduction, 70\% confluent Lewis lung carcinoma cells were incubated with a 1:1 precipitated mixture of pLNCX DsRed-2-retroviralcontaining supernatants of PT67 packaging cells and RPMI 1640 or other culture medium (Life Technologies) containing 10\% fetal bovine serum (Atlanta Biologicals, Norcross, GA) for $72 \mathrm{~h}$ and selected using G418 [35]. 
Nude mice were from AntiCancer Inc. (San Diego, CA). All mouse studies were conducted with an AntiCancer Institutional Animal Care and Use Committee (IACUC)-protocol specifically approved for this study and in accordance with the principals and procedures outlined in the National Institute of Health Guide for the Care and Use of Animals under Assurance Number A3873-1. The animals were observed on a daily basis and humanely sacrificed by $\mathrm{CO}_{2}$ inhalation when they met the following humane endpoint criteria: prostration, skin lesions, significant body weight loss, difficulty breathing, epistaxis, rotational motion and body temperature drop. Animals were housed in a barrier facility on a high efficiency particulate air (HEPA)-filtered rack under standard conditions of 12-hour light/ dark cycles. Animals were housed with no more than 5 per cage. The animals were fed an autoclaved laboratory rodent diet.

\section{Mouse model of aggressive lung cancer}

Mouse tumor models were made by subcutaneously injecting Lewis lung carcinoma cells expressing RFP (LLC-RFP) cells $\left(3 \times 10^{6}\right.$ cells $/ 100 \mu$ PBS $)$ in the flank of nude mice [32].

\section{Preparation of $S$. typhimurium A1-R and VNP20009}

Green fluorescent protein (GFP)-expressing Salmonella typhimurium A1-R bacteria and RFP-labeled VNP20009 were grown overnight in LB medium and then diluted 1:10 in LB medium, respectively. Bacteria were harvested at late-log phase, washed with PBS, and then diluted in PBS.

\section{Comparison of toxicity of $S$. typhimurium A1-R and VNP20009}

Twenty nude mice, 6 weeks old, were used to compare toxicity of S. typhimurium A1-R and VNP20009. The nude mice were randomized into 4 groups of 5 mice. Group 1 mice were treated with $S$. typhimurium A1-R $\left(2.5 \times 10^{7} \mathrm{cfu} / 100 \mu \mathrm{l}\right.$ i.v. $)$, once a week for 5 weeks. Group 2 mice were treated with S. typhimurium A1-R (5 $\times 10^{7} \mathrm{cfu} / 100 \mu 1$ i.v.), once a week for 5 weeks. Group 3 mice were treated with $S$. typhimurium VNP20009 (2.5 $\times 10^{7} \mathrm{cfu} / 100 \mu \mathrm{L}$ i.v.), once a week for 5 weeks. Group 4 mice were treated VNP20009 $\left(5 \times 10^{7} \mathrm{cfu} / 100 \mu \mathrm{L}\right.$ i.v. $)$, once a week for 5 weeks. All mice were used for survival determination for 35 days post initial treatment.
Group 1 mice served as untreated controls. Ten mice in group 2 were treated with $S$. typhimurium A1-R $(1 \times$ $10^{7} \mathrm{cfu} / 100 \mu \mathrm{l}$ i.v.), once a week for 2 weeks. Ten mice in group 3 were treated iv with VNP20009 $\left(1 \times 10^{7} \mathrm{cfu} / 100\right.$ $\mu$ li.v.), once a week for 2 weeks. On the $3^{\text {rd }}$ day after treatment, five mice in each group were sacrificed. Bacteria were isolated from tumors, the liver, spleen and blood and cultured in LB agar. For the remaining five mice in each group, the tumors were removed by surgery to compare with the control group on day 10 after treatment. Tumor weight was determined on day 10 .

\section{Imaging}

Bacteria from the organs were imaged with the Maestro fluorescence imaging system (Perkin-Elmer Inc.). The Maestro multispectral imaging systems contains a liquid crystal tunable filter (LCTF) optically coupled to a CCD camera. Multispectral images are acquired with images typically spaced every $10 \mathrm{~nm}$ throughout the desired spectral rang [36-38].

\section{ACKNOWLEDGEMENT}

This study was supported in part by National Cancer Institute grant CA132971.

\section{Dedication}

This paper is dedicated to the memory of A. R. Moossa, M.D.

\section{Author Contributions}

Conceived and designed the experiments: YZ, RMH, MZ. Performed the experiments: YZ, NZ, MZ. Analyzed the data: YZ, RMH, MZ. Contributed reagents/ materials/analysis tools: RMH, MB. Wrote the manuscript: YZ, RMH, MZ.

\section{REFERENCES}

1. William Coley. Available at: http://en.wikipedia.org/wiki/ William_Coley [last accessed 12 December 2013].

2. Hoffman RM. The preclinical discovery of bacterial therapy for the treatment of metastatic cancer with unique advantages. Expert Opin Drug Discov 2012; 7:73-83.

3. Forbes NS. Engineering the perfect (bacterial) cancer therapy. Nat Rev Cancer 2010; 10:785-94.

4. Hoffman RM. Bugging Tumors. Cancer Discovery 2012; 2 : 588-590. 
5. Yazawa K, Fujimori M, Nakamura T, Sasaki T, Amano J, Kano Y, Taniguchi S. Bifidobacterium longum as a delivery system for gene therapy of chemically induced rat mammary tumors. Breast Cancer Res Treat 2001;66:165-70

6. Dang LH, Bettegowda C, Huso DL, Kinzler KW, Vogelstein B. Combination bacteriolytic therapy for the treatment of experimental tumors. Proc Natl Acad Sci USA 2001;98: 15155-60.

7. Roberts NJ, Zhang L, Janku F, Collins A, Bai RY, Staedtke V, Rusk AW, Tung D, Miller M, Roix J, Khanna KV, Murthy R, Benjamin RS, Helgason T, Szvalb AD, Bird JE, Roy-Chowdhuri S, Zhang HH, Qiao Y, Karim B, McDaniel J, Elpiner A, Sahora A, Lachowicz J, Phillips B, Turner A, Klein MK, Post G, Diaz LA Jr, Riggins GJ, Papadopoulos N, Kinzler KW, Vogelstein B, Bettegowda C, Huso DL, Varterasian M, Saha S, Zhou S. Intratumoral injection of Clostridium novyi-NT spores induces antitumor responses. Sci Transl Med 2014; 6:249ra111.

8. Pawelek JM, Low KB, Bermudes D. Bacteria as tumourtargeting vectors. Lancet Oncol 2003;4:548-556.

9. Pawelek JM, Low KB, Bermudes D. Tumor-targeted Salmonella as a novel anticancer vector. Cancer Res 1997; 57:4537-4544.

10. Toso JF, Gill VJ, Hwu P, Marincola FM, Restifo NP, Schwartzentruber DJ, Sherry RM, Topalian SL, Yang JC, Stock F, Freezer LJ, Morton KE, Seipp C, Haworth L, Mavroukakis S, White D, MacDonald S, Mao J, Sznol M, Rosenberg SA. Phase I study of the intravenous administration of attenuated Salmonella typhimurium to patients with metastatic melanoma. J Clin Oncol 2002; 20:142-52.

11. Zhao M, Yang M, Li X-M, Jiang P, Baranov E, Li S, Xu, M., Penman, S, Hoffman RM. Tumor-targeting bacterial therapy with amino acid auxotrophs of GFP-expressing Salmonella typhimurium. Proc Natl Acad Sci USA 2005; 102:755-760.

12. Zhao M, Yang M, Ma H, Li X, Tan X, Li S, Yang Z, Hoffman RM. Targeted therapy with a Salmonella typhimurium leucine-arginine auxotroph cures orthotopic human breast tumors in nude mice. Cancer Res 2006; 66:7647-7652.

13. Zhao M, Geller J, Ma H, Yang M, Penman S, Hoffman RM. Monotherapy with a tumor-targeting mutant of Salmonella typhimurium cures orthotopic metastatic mouse models of human prostate cancer. Proc Natl Acad Sci USA 2007; 104:10170-10174.

14. Nagakura C, Hayashi K, Zhao M, Yamauchi K, Yamamoto N, Tsuchiya H, Tomita K, Bouvet M, Hoffman RM. Efficacy of a genetically-modifi ed Salmonella typhimurium in an orthotopic human pancreatic cancer in nude mice. Anticancer Res 2009; 29:1873-1878.

15. Hayashi K, Zhao M, Yamauchi K, Yamamoto N, Tsuchiya H, Tomita K, Kishimoto H, Bouvet M, Hoffman RM. Systemic targeting of primary bone tumor and lung metastasis of high-grade osteosarcoma in nude mice with a tumor-selective strain of Salmonella typhimurium. Cell Cycle 2009; 8:870-875.

16. Yam C, Zhao M, Hayashi K, Ma H, Kishimoto H, McElroy M, Bouvet M, Hoffman RM. Monotherapy with a tumortargeting mutant of $S$. typhimurium inhibits liver metastasis in a mouse model of pancreatic cancer. J Surg Res 2010; 164:248-255.

17. Kimura H, Zhang L, Zhao M, Hayashi K, Tsuchiya H, Tomita K, Bouvet M, Wessels J, Hoffman RM. Targeted therapy of spinal cord glioma with a genetically-modified Salmonella typhimurium. Cell Prolif 2010; 43:41-48.

18. Matsumoto Y, Miwa S, Zhang Y, Hiroshima Y, Yano S, Uehara F, Yamamoto M, Toneri M, Bouvet M, Matsubara H, Hoffman RM, Zhao M. Efficacy of tumor-targeting Salmonella typhimurium A1-R on nude mouse models of metastatic and disseminated human ovarian cancer. J Cell Biochem 2014; 115: 1996-2003.

19. Montiyama M, Zhao M, 1 \{jmura H, Tran B, Chishima T, Bouvet M, Endo I, Hoffman RM.Inhibition and eradication of human glioma with tumor-targeting Salmonella typhimurium in an orthotopic nude-mouse model. Cell Cycle 2012; 11: 628-632.

20. Zhang Y, Miwa S, Zhang N, Hoffman RM, Zhao M. Tumor-targeting Salmonella typhimurium A1-R arrests growth of breast-cancer brain metastasis. Oncotarget, in press.

21. Miwa S, Yano S, Zhang Y, Matsumoto Y, Uehara F, Yamamoto M, Hiroshima Y, Kimura H, Hayashi K, Yamamoto N, Bouvet M, Tsuchiya H, Hoffman RM, Zhao M. Tumor-targeting Salmonella typhimurium A1-R prevents experimental human breast cancer bone metastasis in nude mice. Oncotarget 2014; 5: 7119-7125.

22. Liu F, Zhang L, Hoffman RM, Zhao M. Vessel destruction by tumor targeting Salmonella typhimurium A1-R is enhanced by high tumor vascularity. Cell Cycle 2010; 9:4518-4524.

23. Yano S, Zhang Y, Zhao M, Hiroshima Y, Miwa S, Uehara F, Kishimoto H, Tazawa H, Bouvet M, Fujiwara T, Hoffman RM. Tumor-targeting Salmonella typhimurium A1-R decoys quiescent cancer cells to cycle as visualized by FUCCI imaging and become sensitive to chemotherapy. Cell Cycle 2014; 13: 3958-3963.

24. Blagosklonny MV. How cancer could be cured by 2015 . Cell Cycle 2005; 4:269-278.

25. Blagosklonny MV. Tissue-selective therapy of cancer. Br J Cancer 2003; 89:1147-1151.

26. Blagosklonny MV. Matching targets for selective cancer therapy. Drug Discov Today 2003; 8:1104-1107.

27. Blagosklonny MV. "Targeting the absence" and therapeutic engineering for cancer therapy.Cell Cycle 2008; 7:13071312.

28. Blagosklonny MV. Teratogens as anti-cancer drugs. Cell Cycle 2005; 4:1518-1521.

29. Blagosklonny MV. Treatment with inhibitors of caspases, 
that are substrates of drug transporters, selectively permits chemotherapy-induced apoptosis in multidrug-resistant cells but protects normal cells. Leukemia 2001; 15:936-941.

30. Blagosklonny MV. Target for cancer therapy: proliferating cells or stem cells. Leukemia 2006; 20:385-391.

31. Blagosklonny MV. Cancer stem cell and cancer stemloids: from biology to therapy. Cancer Biol Ther 2007; 6:16841690.

32. Hoffman RM, Yang M. Subcellular imaging in the live mouse. Nat Protoc 2006; 1:775-82.

33. Hoffman RM, Yang M. Color-coded fluorescence imaging of tumor-host interactions. Nat Protoc 2006; 1:928-35.

34. Hoffman RM, Yang M. Whole-body imaging with fluorescent proteins. Nat Protoc 2006; 1:1429-1438.

35. Zhao M, Suetsugu A, Ma H, Zhang L, Liu F, Zhang Y, Tran B, Hoffman RM. Efficacy against lung metastasis with a tumor-targeting mutant of Salmonella typhimurium in immunocompetent mice. Cell Cycle 2012; 11: 187-193.

36. Mansfield JR, Gossage KW, Hoyt CC and Levenson RM. Autofluorescence removal, multiplexing, and automated analysis methods for in vivo fluorescence imaging. J Biomed Opt 2005; 10: 41207.

37. Levenson RM and Mansfield JR. Multispectral in biology and medicine: Slices of life. Cytometry A 2006; 69:748758.

38. Zhang Y, Hiroshima Y, Ma H, Zhang N, Hoffman RM, Zhao M. Complementarity of variable-magnification and spectral-separation fluorescence imaging systems for noninvasive detection of metastasis and intravital detection of single cancer cells in mouse models. Anticancer Research, 2015; 35: 661-668. 